\title{
Kinks of plasmid PBR322
}

\author{
L.V. Yakushevich ${ }^{1}$, L.A. Krasnobaeva ${ }^{2,3 *}$ \\ ${ }^{1}$ Institute of Cell Biophysics RAS, Pushchino, Moscow region, Russia \\ ${ }^{2}$ Tomsk State University, Tomsk, Russia \\ ${ }^{3}$ Siberian State Medical University, Tomsk, Russia \\ *e-mail:kla1983@mail.ru
}

Key words: plasmid pBR322, nonlinear dynamics, kinks, McLaughlin-Scott equation

Motivation and Aim: Plasmid pBR322 is a small, circular DNA that is widely used in genetic research, and its components are applied at creation of new instrumental plasmids $[1,2]$. This plasmid has been never investigated theoretically. We suggest, however, that pBR322 is a convenient object for mathematical modeling the internal mobility of the DNA molecule and, in particular, the movement of local conformational distortions bubbles or kinks. In the present paper, the problem of modeling the motion of kinks in the plasmid pBR322, taking into account information on the features of its sequence containing two coding regions: CDS-1 and CDS-2, is posed and solved.

Methods and Algorithms: The movement of kinks in the plasmid pBR322 is investigated by the methods of mathematical modeling. To find the time dependence of the kink velocity and coordinate, we use the method of McLaughlin-Scott [3] complemented by the block method [4] which allows approximately to take into account the dependence of the coefficients of the equation of motion on the sequence of nucleotides. To present the results in a compact and convenient for further analysis form, the $3 \mathrm{D}$ trajectory method has been developed and applied.

Results: The energy profile of plasmid pBR322 has been constructed. It was shown that the kink movement in the plasmid can be interpreted as the movement of a quasi particle in the potential field with the profile having two barriers: CDS-1 and CDS-2. The time dependences of the kink velocity and coordinate were calculated for three values of the initial velocity: 150,1650 and $1879 \mathrm{~m} / \mathrm{s}$. The 3D trajectories of the kinks as well as the projections of these trajectories in the phase plane were constructed.

\section{References}

1. GenBank: https://www.ncbi.nlm.nih.gov/nuccore/J01749.1

2. Watson N. (1988) A new revision of the sequence of plasmid pBR322. Gene. 70(2):399-403.

3. McLaughlin D.W., Scott A.C. (1978) Perturbation analysis of fluxon dynamics. Phys. Rev. A. 18:1652.

4. Yakushevich L.V., Krasnobaeva L.V. (2018) Analytical approaches for exploration of the dynamics of genes having one coding region. Biophysics. 63(1):41-53. 